\title{
SO(2)-CONGRUENT PROJECTIONS OF CONVEX BODIES WITH ROTATION ABOUT THE ORIGIN
}

\author{
BENJAMIN MACKEY \\ (Communicated by Alexander Iosevich)
}

\begin{abstract}
We prove that if two convex bodies $K, L \subset \mathbb{R}^{3}$ satisfy the property that the orthogonal projections of $K$ and $L$ onto every plane containing the origin are rotations of each other, then either $K$ and $L$ coincide or $L$ is the image of $K$ under a reflection about the origin.
\end{abstract}

\section{INTRODUCTION}

In this paper, we will prove the following theorem:

Theorem 1. Let $K, L \subset \mathbb{R}^{3}$ be convex bodies containing the origin as an interior point such that for every $\xi \in S^{2}$, the projection $K_{\mid \xi^{\perp}}$ can be rotated about the origin into $L_{\mid \xi^{\perp}}$. Then either $K=L$ or $K$ can be obtained by reflecting $L$ about the origin.

Several related results have been proven under various assumptions about the type of congruence the projections satisfy. Wilhelm Süss proved that if each projection $K_{\mid \xi^{\perp}}$ is some parallel translation of $L_{\mid \xi^{\perp}}$, then $K$ and $L$ are parallel ([2], page 8). Vladimir Golubyatnikov allowed for both shifts and rotations, and proved two different theorems with variations on the symmetry of the projections ([2], page 13) and smoothness of the bodies ([2, page 22). This new result differs in that no symmetry assumptions are made about the bodies, but the freedom to translate projections is lost.

\section{NotATION AND DEFinitions}

Throughout this paper, $\mathbb{R}^{n}$ will refer to the $n$-dimensional Euclidean space, and $S^{n-1}=\left\{\xi \in \mathbb{R}^{n}:|x|=1\right\}$ will denote the unit sphere. The Euclidean inner product of two vectors $x, y \in \mathbb{R}^{n}$ will be denoted by $x \cdot y$. For a unit vector $\xi \in S^{n-1}$, the hyperplane orthogonal to $\xi$ is denoted by $\xi^{\perp}=\left\{x \in \mathbb{R}^{n}: x \perp \xi\right\}$. The set $\xi^{\perp} \cap S^{2}$ is the great circle of unit vectors orthogonal to $\xi$. If $K \subset \mathbb{R}^{3}$ is a convex body containing the origin and $\xi \in S^{2}$, the section of $K$ orthogonal to $\xi$ is the set $K \cap \xi^{\perp}$. Given $E \subset S^{2}$ endowed with the spherical metric, the interior of $E$ will be denoted by $\operatorname{int}(E)$ and the closure of $E$ will be denoted by $\bar{E}$.

Let $\xi \in S^{2}, r$ be some nonnegative number, and let $x \in \mathbb{R}^{3}$. Then the image of $x$ rotated by an angle of $r \pi$ about the linear subspace spanned by $\xi$ will be denoted

Received by the editors September 18, 2013.

2010 Mathematics Subject Classification. Primary 52A15.

This research was supported in part by the NSF Grant, DMS-1101636. 
by $R_{\xi, r}(x)$. Given $\epsilon>0$, the spherical disk of radius $\epsilon \pi$ centered at $\xi$ in $S^{2}$ will be called $S(\xi, \epsilon)$. We recall some standard concepts in the study of convexity:

Definition 1. Let $\xi \in S^{n-1}$ and $K \subset \mathbb{R}^{n}$ be a convex body. The orthogonal projection of $K$ in the direction $\xi$ is the set $K_{\mid \xi^{\perp}}=\left\{y \in \xi^{\perp}: \exists \lambda \in \mathbb{R}, y+\lambda \xi \in K\right\}$.

Definition 2. Let $K \subset \mathbb{R}^{n}$ be a convex body. The support function of $K$ is the map $h_{K}: S^{n-1} \mapsto \mathbb{R}$ defined by $h_{K}(\xi)=\max \{u \cdot \xi: u \in K\}$. The width function of $K$ is defined by width $h_{K}(\xi)=\left(h_{K}(\xi)+h_{K}(-\xi)\right) / 2$. A body $K$ has constant width if width $_{K}$ is a constant function on $S^{n-1}$.

Definition 3. If $K \subset \mathbb{R}^{n}$ is a convex body, the polar dual of $K$ is the set $K^{*}=\left\{x \in \mathbb{R}^{n}: x \cdot y \leq 1, \forall y \in K\right\}$.

Definition 4. Let $K \subset \mathbb{R}^{n}$ be a convex body containing the origin. The radial function $\rho_{K}: S^{n-1} \mapsto \mathbb{R}$ is defined by $\rho_{K}(\xi)=\max \{\lambda \in \mathbb{R}: \lambda \xi \in K\}$.

\section{Auxillary Results}

For any $r \in \mathbb{R}$, define $F_{r} \subset S^{2}$ by $F_{r}=\left\{\xi \in S^{2}: K_{\mid \xi^{\perp}}\right.$ rotated by $r \pi$ is $\left.L_{\mid \xi^{\perp}}\right\}$. Observe that if a rotation of magnitude between $\pi$ and $2 \pi$ in the clockwise direction is necessary for $K_{\mid \xi^{\perp}}$ to coincide with $L_{\mid \xi^{\perp}}$, then a rotation of less than $\pi$ in the counterclockwise direction makes the projections coincide. Thus, only $r \in[0,1]$ need be considered. It follows that $\xi \in F_{0}$ if and only if $K_{\mid \xi^{\perp}}=L_{\mid \xi^{\perp}}$, and $\xi \in F_{1}$ if and only if the image of $K_{\mid \xi^{\perp}}$ under a reflection about the origin is $L_{\mid \xi^{\perp}}$. The conclusion of Theorem 1 can be rewritten as "either $S^{2}=F_{0}$ or $S^{2}=F_{1}$ ".

Lemma 1. For all $r \in[0,1]$, the set $F_{r}$ is closed.

Proof. If $F_{r}$ is empty, then it is trivially closed. If $F_{r}$ is nonempty, let $\xi_{n}$ be a sequence in $F_{r}$, and suppose $\xi_{n}$ converges to $\xi \in S^{2}$. Let $\theta \in \xi^{\perp}$ be arbitrary. For each $n$, pick some $\theta_{n} \in \xi_{n}^{\perp}$ so that $\theta_{n}$ converges to $\theta$. Since each $\xi_{n} \in F_{r}$, we have $h_{L}\left(R_{\xi_{n}, r}\left(\theta_{n}\right)\right)=h_{K}\left(\theta_{n}\right)$ for each $n$.

By Rodrigues' rotation formula ([3], page 147),

$$
R_{\xi_{n}, r}\left(\theta_{n}\right)=\theta_{n} \cos (r \pi)+\left(\xi_{n} \times \theta_{n}\right) \sin (r \pi)+\xi_{n}\left(\xi_{n} \cdot \theta_{n}\right)(1-\cos (r \pi)) .
$$

Taking the limit as $n$ approaches infinity, we see that $R_{\xi_{n}, r}\left(\theta_{n}\right)$ converges to

$$
\theta \cos (r \pi)+(\xi \times \theta) \sin (r \pi)+\xi(\xi \cdot \theta)(1-\cos (r \pi))=R_{\xi, r}(\theta) .
$$

By the continuity of $h_{K}$ and $h_{L}$, the function $h_{L}\left(R_{\xi_{n}, r}\left(\theta_{n}\right)\right)$ converges to $h_{L}\left(R_{\xi, r}(\theta)\right)$ and $h_{K}\left(\theta_{n}\right)$ converges to $h_{K}(\theta)$. It follows that $h_{L}\left(R_{\xi, r}(\theta)\right)=h_{K}(\theta)$ for every $\theta \in \xi^{\perp}$. This means that $K_{\mid \xi^{\perp}}$ rotated by $r \pi$ coincides with $L_{\mid \xi^{\perp}}$, and so $\xi \in F_{r}$.

Two-dimensional bodies of constant width play an important role in our analysis. Define the set $\Sigma \subset S^{2}$ by $\Sigma=\left\{\xi \in S^{2}: K_{\mid \xi^{\perp}}\right.$ has constant width $\}$. If $\xi_{1}, \xi_{2} \in \Sigma$, then $\xi_{1}^{\perp} \cap S^{2}$ and $\xi_{2}^{\perp} \cap S^{2}$ must intersect, which implies that $K_{\mid \xi_{1}^{\perp}}$ and $K_{\mid \xi_{2}^{\perp}}$ must have the same width, which will be denoted $M$.

Lemma 2. $\Sigma$ is closed.

Proof. Let $\left\{\xi_{n}\right\}_{n=1}^{\infty} \subset \Sigma$ be a sequence such that $\xi_{n}$ converges to $\xi \in S^{2}$, and let $\theta \in \xi^{\perp} \cap S^{2}$ be arbitrary. For each $n$, there is some $\theta_{n} \in \xi_{n}^{\perp}$ so that $\theta_{n}$ converges to $\theta$. Since the width function is continuous, width $h_{K}\left(\theta_{n}\right)$ converges to width $_{K}(\theta)$, but width $_{K}\left(\theta_{n}\right)=M$ for all $M$. Therefore, width $K(\theta)=M$ for all $\theta \in \xi^{\perp} \cap S^{2}$. 
The next lemma is used during the proof of the central lemma in the next section.

Lemma 3. Let $\theta \in S^{2}$ and $\epsilon>0$, and suppose there exists a countable collection of closed subsets $\left\{F_{n}\right\}$ of $S^{2}$ with $\overline{S(\theta, \epsilon)}=\bigcup_{n=1}^{\infty} F_{n}$. Then there exists some $n \in \mathbb{N}$ where $\operatorname{int}\left(F_{n}\right)$ is nonempty.

Proof. The set $\overline{S(\theta, \epsilon)}$ is compact in $S^{2}$ and therefore is a complete metric space. The Baire category theorem (see for example [4 page 98) implies that if $\overline{S(\theta, \epsilon)}=$ $\bigcup_{n=1}^{\infty} F_{n}$, there is some $n \in \mathbb{N}$ with $\operatorname{int}\left(\overline{F_{n}}\right)=\operatorname{int}\left(F_{n}\right) \neq \emptyset$.

\section{MAin Results}

Our strategy in this section will be to combine information about the original bodies and the corresponding dual bodies to reduce the problem to a statement about a system of equations. Solving this system will then reduce further to solving a quartic polynomial equation in one variable. Using this, we will see that any projection which can be rotated by an amount between 0 and $\pi$ into the other projection must be a disk. What this shows is that there actually are no rotations except for the zero rotation and a reflection about the origin.

The following lemma is due to Golubyatnikov ([2, page 17) and is essential to the proof of the main theorem of this paper.

Lemma 4. If the projections of two convex bodies $K, L \subset \mathbb{R}^{3}$ are all $S O(2)$ congruent, then $S^{2}=F_{0} \cup F_{1} \cup \Sigma$.

We will prove a lemma analogous to Lemma 4 which is concerned with the dual bodies $K^{*}$ and $L^{*}$ of $K$ and $L$. The key is that duality takes projections into sections: $\left(K_{\mid \xi^{\perp}}\right)^{*}=K^{*} \cap \xi^{\perp}$ for any $\xi \in S^{2}$ ([1], page 22). This is used in the proof of the following lemma which shows that rotational congruence is inherited by sections of the dual bodies.

Lemma 5. For all $r \in[0,1], F_{r}=\left\{\xi \in S^{2}: K^{*} \cap \xi^{\perp}\right.$ rotated by $r \pi$ is $\left.L^{*} \cap \xi^{\perp}\right\}$.

Proof. If $\xi \in F_{r}$, let $\Phi_{r}: \xi^{\perp} \mapsto \xi^{\perp}$ be the rotation of $\xi^{\perp}$ about the origin by $r \pi$. Since $\Phi_{r}$ is a 1-1 linear transformation such that $\Phi_{r}\left(K_{\mid \xi^{\perp}}\right)=L_{\mid \xi^{\perp}}$, we have $\left(\Phi_{r}\left(K_{\mid \xi^{\perp}}\right)\right)^{*}=\left(L_{\mid \xi^{\perp}}\right)^{*}=L^{*} \cap \xi^{\perp}$. Also, $\left(\Phi_{r}\left(K_{\mid \xi^{\perp}}\right)\right)^{*}=\left(\Phi_{r}^{-1}\right)^{t}\left(K_{\mid \xi^{\perp}}\right)^{*}([1]$, page 21) and $\left(\Phi_{r}^{-1}\right)^{t}=\Phi_{r}$, which implies that $\Phi_{r}\left(K^{*} \cap \xi^{\perp}\right)=L^{*} \cap \xi^{\perp}$.

Define the function $\tau_{K^{*}}: S^{2} \mapsto \mathbb{R}$ by $\tau_{K^{*}}(\xi)=\left(\rho_{K^{*}}^{2}(\xi)+\rho_{K^{*}}^{2}(-\xi)\right) / 2$, and define $\tau_{L^{*}}$ similarly. Lemma 4 is a statement about projections and the width function of $K$, whereas the function $\tau_{K^{*}}$ contains information about the sections of $K^{*}$. We can define the set $\Lambda \subset S^{2}$ analogously to $\Sigma$ by $\Lambda=\left\{\xi \in S^{2}: \tau_{K^{*}}\right.$ restricted to $\xi^{\perp} \cap S^{2}$ is constant $\}$. Observe that if $\xi \in F_{r}$, since the radial function measures distance from the origin, it follows from Lemma 5 that $\tau_{K^{*}}(\theta)=\tau_{L^{*}}\left(R_{\xi, r}(\theta)\right)$ for every $\theta \in \xi^{\perp} \cap S^{2}$.

Lemma 6. $\tau_{K^{*}}(\xi)=\tau_{L^{*}}(\xi)$ for every $\xi \in S^{2}$.

Proof. Since all sections of $K^{*}$ are congruent to corresponding sections of $L^{*}$, area $\left(K^{*} \cap \xi^{\perp}\right)=\operatorname{area}\left(L^{*} \cap \xi^{\perp}\right)$ for every unit vector $\xi$. Since the area of the section can be expressed as $\frac{1}{2} \int_{0}^{2 \pi} \rho_{K^{*} \cap \xi^{\perp}}^{2}(\theta) d \theta$, we can conclude that

$$
\int_{\xi^{\perp} \cap S^{2}} \frac{\rho_{K^{*}}^{2}(\theta)+\rho_{K^{*}}^{2}(-\theta)}{2} d \theta=\int_{\xi^{\perp} \cap S^{2}} \frac{\rho_{L^{*}}^{2}(\theta)+\rho_{L^{*}}^{2}(-\theta)}{2} d \theta
$$


for every $\xi \in S^{2}$. This can be rewritten as

$$
\int_{\xi^{\perp} \cap S^{2}} \tau_{K^{*}}(\theta)-\tau_{L^{*}}(\theta) d \theta=0
$$

for all unit vectors $\xi$. Thus, the spherical Radon transform of the even function $\tau_{K^{*}}-\tau_{L^{*}}$ is identically zero on $S^{2}$, and so (1], page 430) implies that $\tau_{K^{*}}$ and $\tau_{L^{*}}$ coincide everywhere.

Lemma 7. Let $K, L \subset \mathbb{R}^{3}$ be convex bodies containing the origin as an interior point so that for all $\xi \in S^{2}, K_{\mid \xi^{\perp}}$ can be rotated about the origin into $L_{\mid \xi^{\perp}}$. Then $S^{2}=F_{0} \cup F_{1} \cup \Lambda$.

The proof of this lemma closely resembles Golubyatnikov's proof starting on page 17 of [2], and will be postponed until the end of the paper. If we assume that this lemma has been proven, we can complete the proof of Theorem 1 . From Lemma 7 and Lemma 4, we know that if $\xi \in S^{2}$ is not in $F_{0} \cup F_{1}$, then it must be that $\xi \in \Sigma \cap \Lambda$. The main idea is to use duality to show that if $\xi \in \Sigma \cap \Lambda$, then $\xi \in F_{0} \cup F_{1}$, and therefore $S^{2}=F_{0} \cup F_{1}$. Golubyatnikov has proven that this then implies that $S^{2}=F_{0}$ or $S^{2}=F_{1}$ ([2], page 22), which completes the proof of Theorem 1. All that remains is to prove the following corollary.

Corollary 1. $S^{2}=F_{0} \cup F_{1}$.

Proof. By Lemma 4 and Lemma $7 S^{2} \backslash\left(F_{0} \cup F_{1}\right)$ is contained in $\Sigma \cap \Lambda$, so it suffices to prove that $\Sigma \cap \Lambda$ is a subset of $F_{0} \cup F_{1}$. If $\xi \in \Sigma \cap \Lambda$, Lemma 4 implies that there exists a constant $a \in \mathbb{R}$ so that for all $\theta \in \xi^{\perp}$,

$$
h_{K}(\theta)+h_{K}(-\theta)=a .
$$

By Lemma 7, there is a constant $b \in \mathbb{R}$ so that for all $\theta \in \xi^{\perp}$,

$$
\rho_{K^{*}}^{2}(\theta)+\rho_{K^{*}}^{2}(-\theta)=b .
$$

Since $h_{K}(\theta)=1 / \rho_{K^{*}}(\theta)$ for any unit vector $\theta$ ([1], page 20), the second equation can be rewritten as

$$
h_{K}(\theta)^{-2}+h_{K}(-\theta)^{-2}=b
$$

for all $\theta \in \xi^{\perp}$.

Consider the system of equations $x+y=a$ and $x^{-2}+y^{-2}=b$. Since the origin is an interior point of $K$, we can assume that neither $x$ nor $y=a-x$ is equal to zero, and thus this system can be expressed as the quartic equation

$$
(x-a)^{2}+x^{2}=b x^{2}(a-x)^{2},
$$

which has at most four real valued solutions in $x$. For each $\theta \in \xi^{\perp}, h_{K}(\theta)$ is a solution to this equation. Since $h_{K}$ is a continuous function of $\theta$, the intermediate value theorem implies that $h_{K}$ is constant on $\xi^{\perp}$. This implies that both $K_{\mid \xi^{\perp}}$ and $L_{\mid \xi^{\perp}}$ are disks, and thus $K_{\mid \xi^{\perp}}=L_{\mid \xi^{\perp}}$. It follows that $\xi \in F_{0}$, and therefore $\Sigma \cap \Lambda \subset F_{0} \cup F_{1}$.

We conclude this paper by returning to the proof of Lemma 4

Proof of Lemma 17. To prove the lemma, we will show that the set

$$
F=S^{2} \backslash\left(F_{0} \cup F_{1} \cup \Lambda\right)
$$

is empty. An argument similar to the proof of Lemma 2 (just replace $w_{i d t} h_{K}$ with $\left.\tau_{K^{*}}\right)$ shows that $\Lambda$ is closed, so it follows from Lemma 1 and Lemma 5 that $F$ is an 
open set. Suppose there is a unit vector $\xi \in F$ such that $\xi \in F_{r}$ for some irrational $r \in(0,1)$. By Lemma 5 and Lemma 6. we can conclude that for every $\theta \in \xi^{\perp} \cap S^{2}$,

$$
\tau_{K^{*}}(\theta)=\tau_{L^{*}}\left(R_{\xi, r}(\theta)\right)=\tau_{K^{*}}\left(R_{\xi, r}(\theta)\right) .
$$

Fixing some $\theta_{0} \in \xi^{\perp} \cap S^{2}$, an inductive argument shows that

$$
\tau_{K^{*}}\left(R_{\xi, n r}\left(\theta_{0}\right)\right)=\tau_{K^{*}}\left(\theta_{0}\right) \forall n \in \mathbb{N} .
$$

Since $r$ is irrational, the set $\left\{R_{\xi, n r}\left(\theta_{0}\right): n \in \mathbb{N}\right\}$ is dense in the circle $\xi^{\perp} \cap S^{2}$. The function $\tau_{K^{*}}$ is continuous on $S^{2}$, and it takes on a single value on a dense subset of $\xi^{\perp} \cap S^{2}$, so it follows that $\tau_{K^{*}}$ takes a single value on $\xi^{\perp} \cap S^{2}$. Therefore $K^{*} \cap \xi^{\perp}$ is a disk, and thus so is $L^{*} \cap \xi^{\perp}$, which contradicts the assumption that $\xi \notin \Lambda$.

If $F$ is nonempty and none of the sections orthogonal to elements of $F$ coincide after some irrational angle, then $F$ can be rewritten as $F=\bigcup_{r}\left(F \cap F_{r}\right)$ for some subset of the rational numbers contained in $(0,1)$. We claim that there exists some rational $r_{0} \in(0,1)$ with $\operatorname{int}\left(F \cap F_{r_{0}}\right) \neq \emptyset$. To see this, since $F$ is open and assumed nonempty, there exists $\theta \in F$ and $\epsilon>0$ with $\overline{S(\theta, \epsilon)} \subset F$. Then $\overline{S(\theta, \epsilon)}=\cup_{r} \overline{S(\theta, \epsilon)} \cap F_{r}$, and Lemma 3 implies there exists some rational $r_{0} \in(0,1)$ with $\emptyset \neq \operatorname{int}\left(\overline{S(\theta, \epsilon)} \cap F_{r_{0}}\right) \subset \operatorname{int}\left(F \cap F_{r_{0}}\right)$.

Fix some $\xi$ and $\epsilon>0$ with the spherical disk $S(\xi, \epsilon)$ contained in $F \cap F_{r_{0}}$. Then the continuous function $\tau_{K^{*}}$ is not constant along $\xi^{\perp} \cap S^{2}$. Therefore, infinitely many values $c$ exist with corresponding unit vectors $w_{c} \in \xi^{\perp} \cap S^{2}$ so that $\tau_{K^{*}}\left(w_{c}\right)=c$. The rest of the proof will be spent using these values to construct marks on the sphere which are geometrically impossible.

For each value $c=\tau_{K^{*}}\left(w_{c}\right)$ for some $w_{c} \in \xi^{\perp} \cap S^{2}$, denote by $w_{c}^{\prime}$ the vector obtained by rotating $w_{c}$ by $r_{0} \pi$ along $\xi^{\perp} \cap S^{2}$. We claim there is an open arc $l_{c}^{1} \subset S\left(w_{c}, r_{0}\right)$ containing $w_{c}^{\prime}$ with $\tau_{K^{*}}$ identically equal to $c$ on $l_{c}^{1}$. We will then construct another arc $l_{c}^{3}$ on $S^{2}$ which intersects $l_{c}^{1}$ at $w_{c}^{\prime}$ on which $\tau_{K^{*}}$ is also constant, and we will define $X_{c}=l_{c}^{1} \cup l_{c}^{3}$ (see Figure 1).

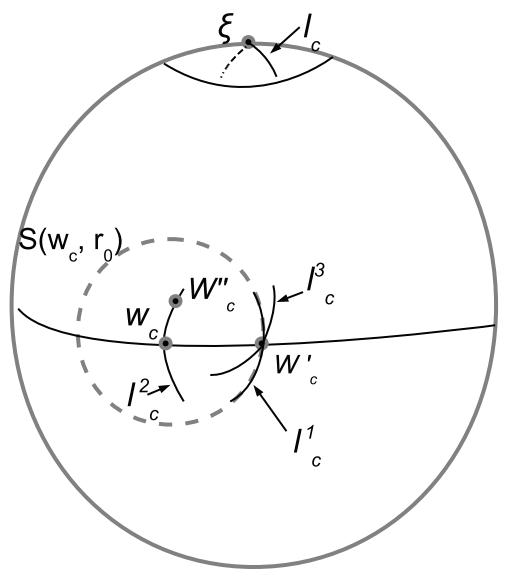

FiguRE 1. The construction of $l_{c}^{1} \cup l_{c}^{3}$ for a clockwise rotation by an angle between 0 and $2 \pi$

Since the spherical disk $S(\xi, \epsilon)$ is contained in $F_{r_{0}}$ and $\xi \in w_{c}^{\perp} \cap S^{2}$, there exists an open arc $l_{c} \subset w_{c}^{\perp} \cap S^{2}$ centered at $\xi$ and contained in $S(\xi, \epsilon) \subset F_{r_{0}}$. For any $v \in l_{c}, v$ is orthogonal to $w_{c}$, and so $w_{c} \in v^{\perp}$, which implies that the great circle 
$v^{\perp} \cap S^{2}$ intersects $S\left(w_{c}, r_{0}\right)$ at the point $R_{v, r_{0}}\left(w_{c}\right)$. Call $l_{c}^{1}=\left\{R_{v, r_{0}}\left(w_{c}\right): v \in l_{c}\right\}$. If $\theta \in l_{c}^{1}$ with $\theta=R_{v, r_{0}}\left(w_{c}\right)$, where $v \in l_{c}$, the fact that $l_{c} \subset F_{r_{0}}$ and Lemma 6 imply that

$$
c=\tau_{K^{*}}\left(w_{c}\right)=\tau_{L^{*}}\left(R_{v, r_{0}}(\theta)\right)=\tau_{L^{*}}(\theta)=\tau_{K^{*}}(\theta),
$$

which proves the claim.

We now construct the second $\operatorname{arc} l_{c}^{3}$. If we start at $w_{c}^{\prime}$ and rotate the projections of $L^{*}$ in the reverse direction, by a similar argument applied to $L^{*}$ we can create $S\left(w_{c}^{\prime}, r_{0}\right)$ and an open arc $l_{c}^{2} \subset S\left(w_{c}^{\prime}, r_{0}\right)$ so that $\tau_{K^{*}}$ takes only the value $c$ on $l_{c}^{2}$. Next, we can pick a third unit vector $w_{c}^{\prime \prime} \in l_{c}^{2}$ distinct from $w_{c}$, and consider the circle $S\left(w_{c}^{\prime \prime}, r_{0}\right)$. Using a similar argument (create an arc in the spherical disk $S(\xi, \epsilon)$ centered at the preimage of $w_{c}^{\prime \prime}$ and consider the image of this arc under the rotation), we can create an $\operatorname{arc} l_{c}^{3} \subset S\left(w_{c}^{\prime \prime}, r_{0}\right)$ on which $\tau_{K^{*}}$ takes only the value $c$. If we define $X_{c}=l_{c}^{1} \cup l_{c}^{3}$ to be the cross mark formed by the two arcs, we see that the function $\tau_{K^{*}}$ takes only the value $c$ on $X_{c}$.

This construction can be done identically for every value taken by $\tau_{K^{*}}$ on $\xi^{\perp} \cap S^{2}$, so we have constructed an infinite family of congruent " $\mathrm{X}$ " figures on the unit sphere. For distinct values $c_{1}, c_{2}$ taken by $\tau_{K^{*}}$ on $\xi^{\perp} \cap S^{2}$, it follows from the construction that $X_{c_{1}}$ and $X_{c_{2}}$ are disjoint. Since it is impossible to construct infinitely many congruent mutually disjoint " $\mathrm{X}$ " figures on the sphere, this completes the proof.

\section{ACKNOWLEDGEMENT}

The author would like to thank Dmitry Ryabogin for helping to organize and revise this paper.

\section{REFERENCES}

[1] Richard J. Gardner, Geometric tomography, 2nd ed., Encyclopedia of Mathematics and its Applications, vol. 58, Cambridge University Press, Cambridge, 2006. MR 2251886 (2007i:52010)

[2] V. P. Golubyatnikov, Uniqueness Questions in Reconstruction of Multidimensional Objects from Tomography-Type Projection Data, VSP, 2000.

[3] Don Koks, Explorations in mathematical physics, Springer, New York, 2006. The concepts behind an elegant language. MR2253604 (2007f:00001)

[4] Walter Rudin, Real and complex analysis, 3rd ed., McGraw-Hill Book Co., New York, 1987. MR 924157 (88k:00002) 48824

Department of Mathematics, Michigan State University, East Lansing, Michigan

E-mail address: mackeybe@msu.edu 